\title{
Soluble ST2 protein in chronic heart failure is independent of traditional factors
}

Karolina Wojtczak-Soska ${ }^{1}$, Tadeusz Pietrucha ${ }^{2}$, Agata Sakowicz², Malgorzata Lelonek ${ }^{1}$

1Department of Cardiology, Medical University of Lodz, Poland

2Department of Medical Biotechnology, Medical University of Lodz, Poland

Submitted: 25 April 2012
Accepted: 22 August 2012

Arch Med Sci 2013; 9, 1: 21-26

DOI: 10.5114 /aoms.2013.33344

Copyright @ 2013 Termedia \& Banach

\author{
Corresponding author: \\ Assoc. Prof. Malgorzata \\ Lelonek MD, PhD \\ Department of Cardiology \\ Medical University of Lodz \\ 1/3 Sterling Str. \\ 91-425 Lodz, Poland \\ Phone: +48 601303401 \\ E-mail: mlelonek@poczta.fm
}

\begin{abstract}
Introduction: ST2 protein is the interleukin 33 (IL-33) receptor, whose serum level depends on the biomechanical strain of cardiac myocytes. The aim of this study was to analyse the relationship between soluble ST2 (SST2) level and traditional factors in patients with chronic heart failure.

Material and methods: Sixty-six patients (mean age 62 years, $75 \%$ males) in stable NYHA class I-III with left ventricular ejection fraction $<45 \%$ were included in the study. Clinical, biochemical, electrocardiographic, echocardiographic and angiographic data were analysed. Patients were divided into groups depending on SST2 median: $>0.28 \mathrm{ng} / \mathrm{ml}(n=31)$ vs. $\leq 0.28 \mathrm{ng} / \mathrm{ml}(n=35)$. SST2 was measured using a quantitative ELISA kit. In order to define factors associated with SST2 levels uni- and multivariate regression analysis was performed.

Results: There was no relationship between SST2 levels and age $(p=0.67)$, body mass index $(p=0.19)$, hsTnT $(p=0.7)$ or other analysed parameters (all $p>0.05$ ), except for N-terminal prohormone B-type natriuretic peptide (NT-proBNP). A significant positive correlation between SST2 and NT-proBNP was found $(p=0.013, R=0.395)$. Multivariate analysis revealed that the stage of coronary artery disease and NT-proBNP were independent factors associated with SST2 concentration $(p=0.04)$. Intriguing is the fact that the fewer the sclerotic changes present in arteries, the higher was the sST2 level $(\beta=-0.381, p=0.04)$. Conclusions: SST2 protein is independent of traditional factors which usually affect levels of NT-proBNP. In chronic heart failure, SST2 protein may be of greater importance in idiopathic dilated cardiomyopathy than in ischaemic aetiology, which seems to be associated with the molecular mechanism (biomechanical strain) related to SST2.
\end{abstract}

Key words: ST2, chronic heart failure, biomarkers.

\section{Introduction}

The use of biomarkers is easy and helps to confirm the diagnosis, optimise the therapy and estimate the prognosis in heart failure (HF) [1]. However, serum concentrations of NT-proBNP, which are now widely used in clinical practice, depend on several factors such as age, sex, left ventricular hypertrophy, tachycardia, right ventricular overload, myocardial ischaemia, hypoxaemia, renal dysfunction, metabolic risk factors, liver cirrhosis, sepsis and infection [1, 2]. Therefore it is necessary to find an independent biomarker.

ST2 protein is an interleukin 33 (IL-33) receptor. The IL-33/ST2 pathway has recently been described as a novel, critical cardioprotective system 
$[3,4]$, which reduces fibrosis and hypertrophy of the myocardium and helps to preserve ventricular function. In chronic HF (CHF) mechanical stress/strain of the left ventricle (LV) wall is exerted on the individual cardiomyocyte, which results in upregulation of ST2 gene expression. Therefore serum concentrations of ST2 soluble form (SST2) depend on mechanical stress [4]. That phenomenon is called biomechanical strain. SST2 is therefore a biomarker of mechanical overload [4, 5]. Myocardial overproduction of ST2, as a result of mechanical strain in $\mathrm{HF}$, leads to the inhibition of IL-33 protective effects. The researchers posed a hypothesis that SST2 is not only a marker of poor prognosis in the case of mechanical ventricular overload, but also a mediator of disease progression. High expectations are associated with this pathway as a target of new HF therapies [3].

SST2 protein is quite well known as an independent prognostic factor for acute HF [6-16]. However, there are only a few studies on SST2 in CHF.

The aim of this study was to determine the relation between SST2 level and traditional clinical, electrocardiographic, biochemical, echocardiographic and angiographic factors assessed during comprehensive evaluation of patients with CHF. It was hypothesized that SST2 protein may be an independent biomarker of CHF, more useful in clinical practice.

\section{Material and methods}

The study enrolled patients in stable NYHA class I-III with LV ejection fraction $<45 \%, 30 \%$ on average (range: $13-44 \%$ ) and receiving optimized therapy. Exclusion criteria were as follows: NYHA class IV, acute coronary syndrome, acute heart failure, inflammatory states, and thyroid dysfunction. Medical history was collected and a 12-lead electrocardiogram was made at hospital entry. Echocardiography and coronary arteriography were performed according to the ASE/EAE and ESC recommendations. Using the M-mode, 2-dimensional and Doppler echocardiographic examinations, left (LA) and right atrial diameter (RA), left ventricular systolic (LVESD) and diastolic dimensions (LVEDD), left ventricular end-systolic (LVESV) and end-diastolic volume (LVEDV), and left ventricular ejection fraction (LVEF) were assessed. Conventional coronary arteriography was performed using a femoral or radial approach. To calculate the sclerotic alteration in coronary arteries we evaluated the degree of luminal obstruction in conventional visual quantification of coronary arteriography.

The following parameters were analysed: demographic and clinical (age, body mass index (BMI), smoking status), electrocardiographic from the standard 12-lead ECG (heart rate, heart rhythm - sinus rhythm or atrial fibrillation, LBBB, QRS duration, QTc), comorbidities (diabetes mellitus, arterial hypertension, stroke, coronary artery disease (CAD)), aetiology of heart failure (ischaemic or nonischaemic - idiopathic dilated cardiomyopathy), basic laboratory tests such as morphology (haemoglobin, white blood cells, neutrophils), sodium levels, estimated glomerular filtration rate (eGFR), high-sensitivity C-reactive protein (hSCRP), highsensitivity troponin $\mathrm{T}$ (hsTnT) and N-terminal prohormone B-type natriuretic peptide (NT-proBNP), echocardiographic variables (LVESD, LVEDD, LA, RA, LVEDV, LVESV, LVEF) and coronary arteriography (stenosis $\geq 50 \%$ of the left main coronary artery and $\geq 75 \%$ of other coronary arteries).

Blood for SST2 level determination was collected at hospital entry and stored at $-76^{\circ} \mathrm{C}$ until analysed. ST2 serum levels were measured using a quantitative ELISA kit. Blood samples $(5 \mathrm{ml})$ were collected into vacuum tubes containing clot activator and after the formation of a clot the samples were centrifuged for $5 \mathrm{~min}$ at $3000 \mathrm{rpm}$. The supernatant (serum) was immediately separated and frozen at $\left(-20^{\circ} \mathrm{C}\right)$. The soluble ST2 level was measured using a sandwich ELISA kit (Medical and Biological Laboratories; Japan). The assay uses two monoclonal antibodies against two different epitopes of human SST2. Serum samples were incubated in microwells coated with the first antihuman SST2 monoclonal antibody. After the washing stage, the second incubation with the peroxidaseconjugated anti-human SST2 monoclonal antibody was conducted. After further washing, the peroxidase substrate was added into each well and the optical density was measured at $450 \mathrm{~nm}$ using a microplate reader.

Patients were divided into groups depending on sST2 median: $>0.28 \mathrm{ng} / \mathrm{ml}(n=31)$ vs. $\leq 0.28 \mathrm{ng} / \mathrm{ml}$ $(n=35)$.

\section{Statistical analysis}

In order to define factors associated with SST2 levels, uni- and multivariate regression analysis was performed. Continuous variables are presented as mean values \pm SD or median values with interquartile ranges (IQR) if not normally distributed. To compare continuous variables with nonnormal distribution the Mann-Whitney $U$ test was performed. Spearman correlation coefficients were used to assess correlations between SST2 and other continuous variables. Associations between nominal variables were examined using Pearson's $\chi^{2}$ test, Yates correction for $\chi^{2}$ test in $2 \times 2$ contingency tables, and the exact Fisher test for larger than $2 \times 2$ contingency tables. Variables which were associated with SST2 level $(p<0.11)$ in the univariate tests were used in the multivariate model. Data were analysed with Statistica software version $8.0(\mathrm{PL})$. A $p$ value $<0.05$ was considered significant. 
The investigation conforms to the principles outlined in the Declaration of Helsinki. The study was approved by the Bioethics Committee and all patients signed an informed consent form.

\section{Results}

This study included 66 patients (mean age: 62 years, $75 \%$ males). Characteristics of the studied population are shown in Table I. The most prevalent comorbidities in the study group were: arterial hypertension (44\%), diabetes mellitus (20\%) and stroke (10\%). In this group, there were 53\% former smokers and $14 \%$ current smokers. Coronary artery disease was demonstrated in $54 \%$ of patients examined. In the studied population the mean concentration of SST2 was $0.29 \mathrm{ng} / \mathrm{ml}$, and the median value was $0.28 \mathrm{ng} / \mathrm{ml}$, with $25^{\text {th }}$ and $75^{\text {th }}$ percentiles of $0.27 \mathrm{ng} / \mathrm{ml}$ and $0.29 \mathrm{ng} / \mathrm{ml}$ respectively.
In this study, significant differences in NT-proBNP $(p=0.018)$ and QRS duration $(p=0.028)$ between groups with different sST2 levels were observed, with higher results in patients with SST2 above the median. Between the studied groups, there were no significant differences in LVEF, hsTnT or other analysed clinical, biochemical, echocardiographic and angiographic parameters, or in the frequency of current smokers $(p=0.5)$, prior myocardial infarction (MI) $(p=0.92)$, and comorbidities such as arterial hypertension $(p=0.88)$, diabetes mellitus $(p=0.11)$ or stroke $(p=0.24)$. Patients' characteristics in both groups depending on SST2 concentration are presented in Table II.

There was no relationship between $\mathrm{SST} 2$ levels and sex $(p=0.75)$, NYHA class $(p=0.12)$, smoking status $(p=0.50)$, presence of comorbidities such as arterial hypertension $(p=0.88)$, diabetes $(p=0.11)$,

Table I. Patient characteristics

\begin{tabular}{|c|c|c|c|c|}
\hline Variables & Mean & Median & Range & SD \\
\hline \multicolumn{5}{|l|}{ Demographic and clinical } \\
\hline Age [years] & 61.970 & 63 & $30-87$ & 11.371 \\
\hline Body mass index $\left[\mathrm{kg} / \mathrm{m}^{2}\right]$ & 26.769 & 26.4 & $16.6-37.1$ & 3.736 \\
\hline Systolic blood pressure [mm Hg] & 116.429 & 115 & $90-190$ & 18.889 \\
\hline Diastolic blood pressure [mm Hg] & 71.032 & 70 & $55-100$ & 10.006 \\
\hline \multicolumn{5}{|l|}{ ECG } \\
\hline Heart rate $[\mathrm{bpm}]$ & 81.048 & 78 & $53-170$ & 22.778 \\
\hline QRS duration [ms] & 106.267 & 100 & $60-180$ & 27.977 \\
\hline QTc [ms] & 385.433 & 380 & $260-509$ & 54.8 \\
\hline \multicolumn{5}{|l|}{ Echocardiographic } \\
\hline LVESD [cm] & 5.522 & 5.4 & $3.4-9.3$ & 1.168 \\
\hline LVEDD $[\mathrm{cm}]$ & 6.683 & 6.5 & $4.6-10.1$ & 1.067 \\
\hline LVESV [ml] & 138.732 & 118.5 & $46-418$ & 71.376 \\
\hline LVEDV [ml] & 195.696 & 180 & $83-490$ & 84.008 \\
\hline $\mathrm{LA}[\mathrm{cm}]$ & 5.941 & 5.850 & $3.6-8$ & 0.866 \\
\hline $\mathrm{RA}[\mathrm{cm}]$ & 5.191 & 5.1 & $3.5-8.1$ & 0.897 \\
\hline LVEF [\%] & 29.477 & 30 & $13-44$ & 7.209 \\
\hline \multicolumn{5}{|l|}{ Biochemical } \\
\hline $\mathrm{sST} 2[\mathrm{ng} / \mathrm{ml}]$ & 0.29 & 0.28 & $0.26-0.59$ & 0.02 \\
\hline NT-proBNP [mg/dl] & 4043.533 & 2279 & $25.48-15600$ & 4278.951 \\
\hline hsTnT [mg/dl] & 0.063 & 0.026 & $0.004-0.36$ & 0.085 \\
\hline Creatinine [mg/dl] & 1.051 & 1 & $0.5-1.9$ & 0.283 \\
\hline hsCRP $[\mathrm{mg} / \mathrm{dl}]$ & 14.115 & 5.9 & $0.4-157$ & 28.370 \\
\hline Sodium [mmol/l] & 137.088 & 138 & $127-144$ & 3.622 \\
\hline eGFR [ml/min/1.73 m²] & 83.369 & 80 & $27-185$ & 31.032 \\
\hline WBC $\left[10^{3} / \mu \mathrm{l}\right]$ & 7.44 & 7.4 & $3.36-12.7$ & 1.99 \\
\hline Neutrophils [\%] & 61.667 & 60.95 & $28.8-81.3$ & 10.133 \\
\hline Haemoglobin [mg/dl] & 14.334 & 14.3 & $9.2-18.8$ & 1.887 \\
\hline
\end{tabular}


Table II. Baseline characteristics by SST2 median

\begin{tabular}{|c|c|c|c|}
\hline Variables & sST2 $\leq$ Median & sST2 > Median & Value of $p$ \\
\hline \multicolumn{4}{|l|}{ Demographic and clinical } \\
\hline Age [years] & $61.7 \pm 12.2$ & $62.2 \pm 10.4$ & 0.87 \\
\hline Female, $n$ & 7 & 5 & 0.75 \\
\hline Ischaemic HF, $n$ & 20 & 15 & 0.55 \\
\hline Body mass index $\left[\mathrm{kg} / \mathrm{m}^{2}\right]$ & $25.9 \pm 3.6$ & $27.7 \pm 3.6$ & 0.12 \\
\hline Systolic blood pressure [mm Hg] & $117 \pm 21.8$ & $115 \pm 14.9$ & 0.92 \\
\hline Diastolic blood pressure [mm Hg] & $71.3 \pm 10.1$ & $70.6 \pm 9.9$ & 0.83 \\
\hline \multicolumn{4}{|l|}{ ECG } \\
\hline Heart rate $[\mathrm{bpm}]$ & $79 \pm 21$ & $83.2 \pm 24.8$ & 0.35 \\
\hline QRS duration [ms] & $98.7 \pm 25.8$ & $114.8 \pm 28.2$ & 0.02 \\
\hline QTc [ms] & $388 \pm 51.6$ & $382 \pm 58.9$ & 0.81 \\
\hline \multicolumn{4}{|l|}{ Echocardiographic } \\
\hline LVESD [cm] & $5.4 \pm 1.1$ & $5.6 \pm 1.2$ & 0.75 \\
\hline LVEDD [cm] & $6.7 \pm 1$ & $6.7 \pm 1.1$ & 0.94 \\
\hline LVESV [ml] & $134.8 \pm 71$ & $142.9 \pm 72$ & 0.66 \\
\hline LVEDV [ml] & $190.8 \pm 7634$ & $200.9 \pm 92.6$ & 0.69 \\
\hline LA [cm] & $5.8 \pm 0.9$ & $6 \pm 0.8$ & 0.11 \\
\hline $\mathrm{RA}[\mathrm{cm}]$ & $5.1 \pm 0.9$ & $5.2 \pm 0.8$ & 0.56 \\
\hline LVEF [\%] & $30.6 \pm 8.7$ & $28.9 \pm 6.2$ & 0.28 \\
\hline \multicolumn{4}{|l|}{ Coronarography (extent of CAD) } \\
\hline 1 vessel & 8 & 5 & 0.50 \\
\hline 2 vessels & 7 & 2 & 0.13 \\
\hline 3 vessels & 2 & 4 & 0.40 \\
\hline \multicolumn{4}{|l|}{ Biochemical } \\
\hline NT-proBNP [mg/dl] & $2675 \pm 3181$ & $5791.6 \pm 4920$ & 0.01 \\
\hline hsTnT [mg/dl] & $0.069 \pm 0.11$ & $0.057 \pm 0.051$ & 0.63 \\
\hline Creatinine $[\mathrm{mg} / \mathrm{dl}]$ & $1.05 \pm 0.23$ & $1.05 \pm 0.33$ & 0.51 \\
\hline hsCRP [mg/dl] & $12.2 \pm 20.8$ & $16.06 \pm 35.04$ & 0.95 \\
\hline Sodium $[\mathrm{mmol} / \mathrm{l}]$ & $137 \pm 3.2$ & $136 \pm 4$ & 0.29 \\
\hline $\mathrm{eGFR}\left[\mathrm{ml} / \mathrm{min} / 1.73 \mathrm{~m}^{2}\right]$ & $79.5 \pm 28.2$ & $87.8 \pm 33.9$ & 0.41 \\
\hline WBC $\left[10^{3} / \mu \mathrm{l}\right]$ & $10.1 \pm 1.5$ & $7.5 \pm 2.2$ & 0.59 \\
\hline Neutrophils [\%] & $61.2 \pm 10.7$ & $62.3 \pm 9.4$ & 0.90 \\
\hline Haemoglobin [mg/dl] & $14.2 \pm 1.5$ & $14.4 \pm 2.2$ & 0.30 \\
\hline
\end{tabular}

stroke $(p=0.24)$ and other variables presented in Table III, except for NT-proBNP. A moderate positive correlation between SST2 and NT-proBNP $(R=0.34$, $p=0.013)$ was found. Moreover, multivariate regression analysis revealed that the number of atherosclerotic coronary arteries and NT-proBNP were independent factors associated with SST2 concentration $(p=0.04)$. The fewer the sclerotic changes present in arteries and the higher the NT-proBNP level, the higher was the sST2 level $(\beta=-0.381, p=0.04)$. The above results are presented in Figure 1 .

\section{Discussion}

The main finding of this study is the observed association between SST2 and the number of atherosclerotic coronary arteries. To the best of our knowledge, this study is the first to reveal such a relationship. Our result is consistent with the role of the IL-33/ST2 pathway in inflammatory diseases, including atherosclerosis. ST2 protein inhibits the proinflammatory effect of IL-33 [17, 18]. We hypothesise that higher concentrations of SST2 in primary 
non-ischaemic HF could be associated with the inhibition of atherosclerosis progression in coronary arteries. The observed relationship could also be helpful in the understanding of the molecular mechanism related to SST2 production by cardiac fibroblasts and cardiomyocytes (biomechanical strain). Usually regional contractile dysfunction in ischaemic HF and generalised contractile dysfunction in non-ischaemic HF may influence the process of SST2 production. In contrast to our findings, Shimpo et al. [11] observed no correlation between SST2 levels and extent of CAD; however, their research enrolled patients with $\mathrm{MI}$, not with $\mathrm{CHF}$ as in our study.

The second important finding is that SST2 is a biomarker independent of traditional risk factors (clinical, echocardiographic and electrocardiographic) that are potentially related to the progression of CHF. Until now, there have been no studies concerning the relationships between $\mathrm{SST} 2$ and factors assessed during comprehensive evaluation in CHF. However, some studies revealed an association between SST2 and LVEF in acute and destabilised $\mathrm{HF}[8,10,14]$ and also in non-ischaemic HF [19]. A weak correlation between SST2 and hsTnT was also reported in patients with $\mathrm{MI}$ [11].

In our study, traditional clinical features such as age, sex and BMI did not correlate with SST2 level. This finding is in agreement with other studies concerning acute $[8,10,11]$ and chronic HF [19]. One of them demonstrated that in patients with MI sST2 protein is independent of clinical characteristics such as age, sex or previous MI [8]. Also Shah et al. [16] found that SST2 protein in acute HF is independent of the traditional biochemical and echocardiographic parameters. Moreover, Weinberg et al. [19] in a study of 161 patients with severe CHF demonstrated that baseline SST2 was similar in female and male patients $(p=0.36)$, and a difference in SST2 level observed 2 weeks after enrolment was not affected by sex $(p=0.55)$.

We found a correlation between SST2 protein and NT-proBNP, which is consistent with previous results concerning acute $[8,10,15]$ and chronic $\mathrm{HF}$ $[19,20]$. Our study revealed that higher concentrations of SST2 were associated with wider QRS. We did not find any similar reports in the literature. It is well known that QRS duration is associated with left ventricle failure. SST2 levels depend on the biomechanical strain which is observed in impaired LV. Therefore a correlation between QRS duration and sST2 is possible.

Further studies of SST2 as an independent biomarker should be conducted among CHF patients, in order to assess its prognostic utility. International research centres have investigated the role of the protein $\mathrm{SST} 2$ as a useful factor in the identification of patients at increased risk of sudden cardiac
Table III. Spearman rank order correlations between SST2 and other variables

\begin{tabular}{|c|c|c|}
\hline Variables & Value of $p$ & $R$ \\
\hline \multicolumn{3}{|l|}{ Demographic and clinical } \\
\hline Age & 0.665 & 0.06 \\
\hline Body mass index & 0.189 & 0.18 \\
\hline Systolic blood pressure & 0.853 & 0.02 \\
\hline Diastolic blood pressure & 0.386 & 0.02 \\
\hline \multicolumn{3}{|l|}{ ECG } \\
\hline Sinus rhythm & 0.164 & -0.18 \\
\hline Heart rate & 0.609 & 0.07 \\
\hline QRS duration & 0.174 & 0.18 \\
\hline QTC & 0.574 & 0.08 \\
\hline \multicolumn{3}{|l|}{ Echocardiographic } \\
\hline LVESD & 0.584 & -0.08 \\
\hline LVEDD & 0.503 & -0.09 \\
\hline LVESV & 0.540 & -0.06 \\
\hline LVEDV & 0.650 & -0.06 \\
\hline LA & 0.420 & 0.11 \\
\hline RA & 0.878 & 0.02 \\
\hline LVEF & 0.889 & 0.02 \\
\hline \multicolumn{3}{|l|}{ Biochemical } \\
\hline NT-proBNP & 0.012 & 0.39 \\
\hline hsTnT & 0.703 & -0.1 \\
\hline Creatinine & 0.552 & -0.07 \\
\hline hsCRP & 0.645 & 0.07 \\
\hline Sodium & 0.065 & -0.25 \\
\hline eGFR & 0.670 & 0.06 \\
\hline WBC & 0.864 & 0.02 \\
\hline Neutrophils & 0.339 & 0.13 \\
\hline Haemoglobin & 0.361 & 0.12 \\
\hline
\end{tabular}

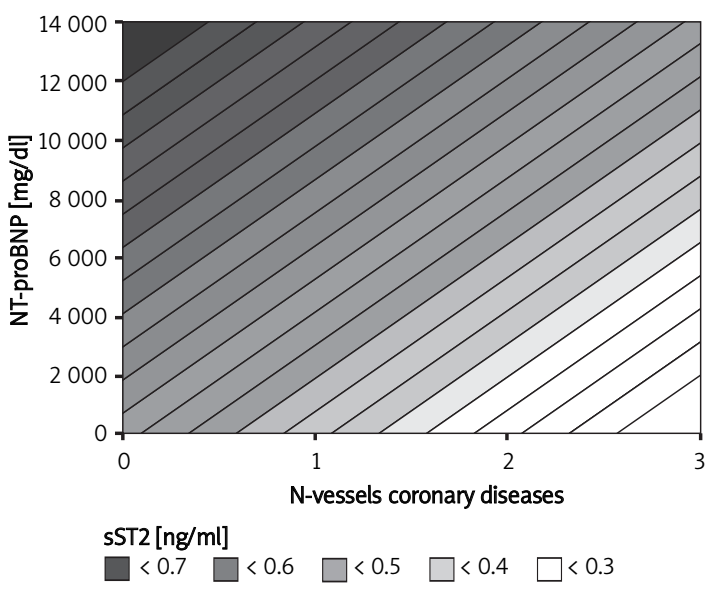

Figure 1. Diagram of relationship between SST2 level, NT-proBNP level and the number of atherosclerotic coronary arteries 
death, in acute HF and acute coronary syndromes [6-16]. Recently, some studies concerning the role of SST2 in the assessment of CHF prognosis have been performed [20-23].

Our study has limitations. The main limitation is the small number of patients. There is a need for further research conducted on a larger population. Quantification and detection of coronary artery stenoses were done under conventional visual evaluation without an algorithm standardized methodology such as the Rotterdam Coronary Artery Algorithm Evaluation Framework.

In conclusion, sST2 protein is independent of traditional factors, such as age and BMI, which usually affect levels of NT-proBNP. In chronic heart failure, SST2 protein may be of greater importance in idiopathic dilated cardiomyopathy than in ischaemic aetiology of HF, which seems to be associated with the molecular mechanism (biomechanical strain) related to SST2.

\section{Acknowledgments}

This work was supported by a Medical University of Lodz grant (502-03/1-005-02/502-14-090) to Karolina Wojtczak-Soska.

For English correction we thank Monika Zelazowska MD, PhD (Austin, Texas).

\section{References}

1. Dickstein K, Cohen-Solal A, Filippatos G, et al. ESC Guidelines for the diagnosis and treatment of acute and chronic heart failure 2008. Eur Heart J 2008; 29: 2388-442.

2. Bao $Y$, Shang $X$, Zhou L, et al. Relationship between $\mathrm{N}$-terminal pro-B-type natriuretic peptide levels and metabolic syndrome. Arch Med Sci 2011; 7: 247-56.

3. Kakkar R, Lee RT. The IL-33/ST2 pathway: therapeutic target and novel biomarker. Nat Rev Drug Discov 2008; 7: 827-40.

4. Sanada S, Hakuno D, Higgins LJ, Schreiter ER, McKenzie AN, Lee RT. IL-33 and ST2 comprise a critical biomechanically induced and cardioprotective signaling system. J Clin Invest 2007; 117: 1538-49.

5. Bhardwaj A, Januzzi JL Jr. ST2: a novel biomarker for heart failure. Expert Rev Mol Diagn 2010; 10: 459-64.

6. Mueller T, Dieplinger B, Gegenhuber A, Poelz W, Pacher R, Haltmayer M. Increased plasma concentrations of soluble ST2 are predictive for 1-year mortality in patients with acute destabilized heart failure. Clin Chem 2008; 54 752-6.

7. Boisot S, Beede J, Isakson S, et al. Serial sampling of ST2 predicts 90-day mortality following destabilized heart failure. J Card Fail 2008; 14: 732-38.

8. Sabatine MS, Morrow DA, Higgins LJ, et al. Complementary roles for biomarkers of biomechanical strain ST2 and N-terminal prohormone B-type natriuretic peptide in patients with ST-elevation myocardial infarction. Circulation 2008; 117: 1936-44.

9. Bayes-Genis A, Pascual-Figal D, Januzzi JL, et al. Soluble ST2 monitoring provides additional risk stratification for outpatients with decompensated heart failure. Rev Esp Cardiol 2010; 63: 1171-8.
10. Rehman SU, Mueller T, Januzzi JL Jr. Characteristics of the novel interleukin family biomarker ST2 in patients with acute heart failure. J Am Coll Cardiol 2008; 52: 1458-65.

11. Shimpo M, Morrow DA, Weinberg EO, et al. Serum levels of the interleukin-1 receptor family member ST2 predict mortality and clinical outcome in acute myocardial infarction. Circulation 2004; 109: 2186-90.

12. Eggers KM, Armstrong PW, Califf RM, et al. ST2 and mortality in non-ST-segment elevation acute coronary syndrome. Am Heart J 2010; 159: 788-94.

13. Chan D, Ng LL. Biomarkers in acute myocardial infarction. BMC Med 2010; 8: 34-44.

14. Manzano-Fernandez S, Mueller T, Pascual-Figal D, Truong QA, Januzzi JL. Usefulness of soluble concentrations of interleukin family member ST2 as predictor of mortality in patients with acutely decompensated heart failure relative to left ventricular ejection fraction. Am J Cardiol 2011; 107: 259-67.

15. Pascual-Figal D, Manzano-Fernandez S, Boronat M, et al. Soluble ST2, high-sensitivity troponin $\mathrm{T}$ - and $\mathrm{N}$-terminal pro-B-type natriuretic peptide: complementary role for risk stratification in acutely decompensated heart failure. Eur J Heart Fail 2011; 13: 718-25.

16. Shah RV, Januzzi JL Jr. ST2: a novel remodeling biomarker in acute and chronic heart failure. Curr Heart Fail Rep 2010; 7: 9-14.

17. Oshikawa K, Yanagisawa K, Tominaga S, Sugiyama Y. Expression and function of the ST2 gene in a murine model of allergic airway inflammation. Clin Exp Allergy 2002; 32: 1520-6.

18. Leung BP, Xu D, Culshaw S, McInnes IB, Liew FY. A novel therapy of murine collagen-induced arthritis with soluble T1/ST2. J Immunol 2004; 173: 145-50.

19. Weinberg EO, Shimpo M, Hurwitz S, Tominaga S, Rolueau J, Lee RT. Identification of serum soluble ST2 receptor as a novel heart failure biomarker. Circulation 2003; 107: 721-6.

20. Pascual-Figal D, Ordonez-Llanos Jtornel PL, Vazquez R, et al. Soluble ST2 for predicting sudden cardiac death in patients with chronic heart failure and left ventricular systolic dysfunction. J Am Coll Cardiol 2009; 54: 2174-9.

21. Daniels LB, Clopton P, Iqbal N, Tran K, Maisel AS. Association of ST2 levels with cardiac structure and function and mortality in outpatients. Am Heart J 2010; 160: 721-8.

22. Ky B, French B, McCloskey K, et al. High-sensitivity ST2 for prediction of adverse outcomes in chronic heart failure. Circ Heart Fail 2011; 4: 180-7.

23. Ky B, French B, Levy WC, et al. Multiple biomarkers for risk prediction in chronic heart failure. Circ Heart Fail 2012; 5: 183-90. 